\title{
La reducción farmacológica de la presión arterial es efectiva para prevenir eventos cardiovasculares y muertes en todos los grupos de edad
}

Pharmacological blood pressure reduction is effective in preventing cardiovascular events and deaths in all age groups

\section{Comentado de}

Blood Pressure Lowering Treatment Trialists' Collaboration. Lancet. 2021 Sep 18;398(10305):1053-1064. DOI: 10.1016/S0140-6736(21)01921-8. PMID: 344610401

\section{Objetivo}

Investigar el efecto estratificado del tratamiento farmacológico antihipertensivo en el riesgo de desarrollar eventos cardiovasculares y muerte de acuerdo a la edad, la tensión arterial sistólica (TAS) y la tensión arterial diastólica (TAD) al inicio del estudio.

\section{Diseño}

Meta-análisis de datos de pacientes individuales.

\section{Fuentes de datos}

Información de 52 ensayos clínicos aleatorizados (ECA) provista por la Colaboración de Ensayos Clínicos en Tratamientos para la Disminución de la Presión Arterial (Blood Pressure Lowering Treatment Trialists' Collaboration, BPLTTC). Además, los autores realizaron búsquedas en MEDLINE.

\section{Selección de estudios}

Fueron elegibles los ECAs que evaluaron tratamientos farmacológicos antihipertensivos versus placebo, o estrategias de tratamiento más intensivas versus menos intensivas, con al menos 1000 personas-año de seguimiento en cada grupo, y que proveyeran información sobre los desenlaces analizados, incluyendo el tipo y el momento de los eventos, la edad y las mediciones iniciales de presión arterial. Fueron excluidos los participantes con antecedentes de insuficiencia cardíaca.

\section{Medición de resultados}

El desenlace primario, denominado eventos cardiovasculares mayores, fue definido como una combinación de accidente cerebrovascular (ACV) fatal o no fatal, infarto de miocardio (IM) cardiopatía isquémica fatal o no fatal, o insuficiencia cardíaca fatal o que requiriese internación. Los desenlaces secundarios fueron la mortalidad por cualquier causa y cada componente del desenlace primario.

Los pacientes fueron agrupados según su edad al inicio del estudio (<55 años, 55 a 64 años, 65 a 74 años, 75 a 84 años y $\geq 85$ años), y por nivel de presión arterial, en siete categorías de TAS (de $<120 \mathrm{mmHg}$ a $\geq 170 \mathrm{mmHg}$ ) y seis categorías de TAD (de $<70 \mathrm{mmHg}$ a $\geq 110 \mathrm{mmHg}$ ) con incrementos de $10 \mathrm{mmHg}$ en cada una de ellas.

El riesgo de sesgo fue evaluado mediante la herramienta Cochrane.

\section{Resultados Principales}

El meta-análisis fue realizado sobre 51 ECAs con un total de 358.707 pacientes. Los participantes tenían entre 21 y 105 años de edad (mediana 65 años, intervalo intercuartilo [IIC] 59 a 75). La mayor mediana de tiempo de seguimiento se observó entre los participantes de 55 años o menos $(4,5$ años, IIC 3,1) y la menor, entre los de 85 años o más (2,8 años).

La Tabla 1 presenta los resultados sobre los eventos cardiovasculares de la reducción de $5 \mathrm{mmHg}$ en la TAS por grupo de edad. Fueron observados patrones similares en la reducción de riesgo de estos eventos con una disminución de $3 \mathrm{mmHg}$ en la TAD. Las reducciones en el riesgo absoluto (RRA) de eventos cardiovasculares mayores fueron variables según la edad, y con mayor beneficio en los grupos de edad más avanzada ( $p$-valor de interacción ajustada $=0,024$ )

Al analizar el riesgo relativo (RR) de manera desagregada por edad y por valores de TAS o TAD inicial, los autores no encontraron un patrón claro de efectos proporcionales crecientes entre los individuos con presión arterial sistólica o diastólica basal más alta sobre el riesgo de eventos cardiovasculares importantes o la mortalidad.

Por otro lado, las pruebas de heterogeneidad no indicaron inconsistencia significativa en los efectos relativos del tratamiento para las diferentes categorías de presión arterial inicial en ningún grupo etario.

Tabla 1. Riesgo relativo y reducción del riesgo absoluto por cada $5 \mathrm{mmHg}$ de descenso de la presión arterial sistólica en la incidencia de eventos cardiovasculares mayores, estratificado por edad. Nota: IC: intervalo de confianza.

\begin{tabular}{|l|c|c|c|c|}
\hline $\begin{array}{l}\text { Grupo de } \\
\text { Edad }\end{array}$ & $\begin{array}{c}\text { Grupo Intervención, N de } \\
\text { eventos/Total }\end{array}$ & $\begin{array}{c}\text { Grupo Comparación, } N \text { de } \\
\text { eventos/Total }\end{array}$ & $\begin{array}{c}\text { Cociente de Riesgo (IC } \\
95 \%)\end{array}$ & $\begin{array}{c}\text { Reducción del Riesgo Absoluto (IC } \\
95 \%)\end{array}$ \\
\hline$<55$ años & $1.485 / 21.594$ & $1.742 / 20.731$ & $0,82(0,76$ a 0,88$)$ & $-0,015(-0,020$ a $-0,010)$ \\
\hline 55 a 64 años & $5.636 / 59.649$ & $7.080 / 67.526$ & $0,91(0,88$ a 0,95$)$ & $-0,010(-0,014$ a $-0,007)$ \\
\hline 65 a 74 años & $7.413 / 59.560$ & $9.551 / 67.934$ & $0,91(0,88$ a 0,95$)$ & $-0,016(-0,020$ a $-0,012)$ \\
\hline 75 a 84 años & $3.825 / 24.747$ & $5.174 / 28.944$ & $0,91(0,87$ a 0,96$)$ & $-0,024(-0,031$ a $-0,017)$ \\
\hline$\geq 85$ años & $459 / 2.247$ & $582 / 2.528$ & $0,99(0,87$ a 1,12$)$ & $-0,026(-0,052$ a 0,001$)$ \\
\hline
\end{tabular}




\section{Conclusiones}

Este estudio mostró que la reducción farmacológica de la presión arterial fue efectiva en un amplio rango de edades, sin evidencia de que las reducciones del RR para la prevención de eventos cardiovasculares mayores varíen según los niveles de presión arterial sistólica o diastólica al momento de la aleatorización, hasta menos de 120/70 mmHg. Aunque los autores encontraron evidencia de una disminución de las reducciones del RR con el aumento de la edad, las RRA no siguieron el mismo patrón y parecieron ser mayores en los grupos de mayor edad. Los efectos estratificados sobre la mortalidad por todas las causas siguieron un patrón similar, sin evidencia que sugiera que el tratamiento aumentara la mortalidad en ninguno de los grupos etarios.

Por lo tanto, la reducción farmacológica de la presión arterial debe considerarse una opción terapéutica importante de manera independiente de la edad, con la eliminación de los umbrales de presión arterial según grupos etarios de las guías internacionales.

Fuente de financiamiento / Conflicto de interés de los autores: British Heart Foundation, NIHR Oxford Biomedical Research Center y Oxford Martin School. Algunos de los autores declararon recibir subsidios u honorarios de entidades públicas o privadas (detalladas en el artículo original).

\section{Comentario}

En los últimos años, las guías de práctica clínica han virado hacia recomendaciones más intensivas de tratamiento de la hipertensión arterial (HTA), en términos del momento de inicio de la terapia farmacológica, el número de drogas utilizadas y las metas terapéuticas ${ }^{2-4}$. Sin embargo, persiste el debate sobre cuál debería ser el enfoque para el tratamiento de la HTA en los adultos mayores. La preocupación por una peor tolerancia o una mayor incidencia de efectos adversos con la terapia farmacológica han constituido barreras para el tratamiento de la HTA en esta población. El estudio resumido ${ }^{1}$ coincide con otros artículos recientes que evidencian que el tratamiento farmacológico antihipertensivo reduce la morbimortalidad asociada a la HTA incluso en edades avanzadas ${ }^{5,6}$.

De todas maneras, es importante tener en cuenta que los estudios no incluyen de manera habitual a los pacientes denominados frágiles - aquellos con una disminución de las reservas fisiológicas y un mayor riesgo de declinar, lo que los sitúa en una situación de mayor vulnerabilidad ante perturbaciones externas y una mayor probabilidad para presentar episodios adversos de salud (hospitalización, institucionalización, muerte, caídas) y pérdida de función, discapacidad o dependencia ${ }^{7}$. Además, no deja de ser cierto que los pacientes mayores suelen tener otras comorbilidades como insuficiencia renal, enfermedad vascular, hipotensión postural, etc., y suelen tomar otros medicamentos que pueden interactuar negativamente con ciertas drogas antihipertensivas. A pesar de esto, la edad como factor único no debería ser una barrera para el tratamiento farmacológico adecuado de la HTA.

\section{Conclusiones de la comentadora}

Este estudio proporciona evidencia de que, incluso en individuos de más de 65 años de edad, la reducción de la presión arterial es efectiva para prevenir la ocurrencia de eventos cardiovasculares y la mortalidad. Si bien este resultado fue marginalmente no significativo entre los pacientes de 85 años o más, continúa reforzando el concepto de que la edad, considerada de manera aislada, no debería ser un factor que limite la terapia antihipertensiva en los adultos mayores. Las decisiones sobre el mejor tratamiento antihipertensivo para los pacientes mayores con HTA deben ser tomadas en forma compartida, eligiendo los fármacos apropiados para cada paciente en particular (considerando factores como su perfil de riesgo, sus comorbilidades y su medicación previa) y supervisando la terapia de manera más intensiva.

M. Victoria Salgado [ Centro de Estudios de Estado y Sociedad; Servicio de Medicina Familiar, Hospital de Alta Complejidad SAMIC El Calafate; Departamento de Medicina, Instituto Universitario Hospital Italiano de Buenos Aires. victoria.salgado@cedes.org ]

Salgado MV La reducción farmacológica de la presión arterial es efectiva para prevenir eventos cardiovasculares y muertes en todos los grupos de edad. Evid Actual Pract Ambul. 2022;25(1):e007000. Available from: https://dx.doi.org/10.51987/EVIDENCIA.V25I1.7000. Comentado de: Blood Pressure Lowering Treatment Trialists' Collaboration. Age-stratified and blood-pressure-stratified effects of blood-pressure-lowering pharmacotherapy for the prevention of cardiovascular disease and death: an individual participant-level data meta-analysis. Lancet. 2021 Sep 18;398(10305):1053-1064. doi: 10.1016/S0140-6736(21)01921-8. PMID: 34461040

\section{Referencias}

1. Blood Pressure Lowering Treatment Trialists' Collaboration. Age-stratified and blood-pressure-stratified effects of blood-pressure-lowering pharmacotherapy for the prevention of cardiovascular disease and death: an individual participant-level data meta-analysis. Lancet. $2021 ; 398: 1053-64$. Available from: 10.1016/S0140-6736(21)01921-8.

2. Williams B, Mancia G, Spiering W, et al. ESC/ESH Guidelines for the management of arterial hypertension. Eur Heart J. 2018;39:3021-3104. Available from: 10.1093/eurheartj/ehy339.

3. World Health Organization. Guideline for the pharmacological treatment of hypertension in adults; 2021. Available from: https://apps.who.int/iris/ handle/10665/344424.

4. Unger T, Borghi C, Charchar F, et al. 2020 International Society of Hypertension Global Hypertension Practice Guidelines. Hypertension. 2020;75:1334-57. Available from: 10.1161/HYPERTENSIONAHA.120.15026.

5. Briasoulis A, Agarwal V, Tousoulis D, et al. Effects of antihypertensive treatment in patients over 65 years of age: a meta-analysis of randomised controlled studies. Heart. 2014;100:317-340. Available from: 10.1136/heartjnl-2013-304111.

6. Beckett NS, Peters R, Fletcher AE, et al. Treatment of hypertension in patients 80 years of age or older. N Engl J Med. 2008;358:1887-98. Available from: 10.1056/NEJMoa0801369.

7. Lesende IM, Gorroñogoitia-Iturbe A, Pavón JG, et al. El anciano frágil. Detección y tratamiento en Atención Primaria. Aten Primaria. 2010;42(7):38893. Available from: 10.1016/j.aprim.2009.09.022. 